\title{
Extensive demethylation of normally hypermethylated CpG islands occurs in human atherosclerotic arteries
}

\author{
SILVIA A. CASTILLO-DÍAZ ${ }^{1}$, MARÍA E. GARAY-SEVILLA² ${ }^{2}$ MARTHA A. HERNÁNDEZ-GONZÁLEZ³ \\ MARTHA O. SOLÍS-MARTÍNEZ ${ }^{2}$ and SILVIO ZAINA ${ }^{2}$ \\ Departments of ${ }^{1}$ Medicine and Nutrition and ${ }^{2}$ Medical Sciences, Division of Health Sciences, Leon Campus, \\ University of Guanajuato, 20 de Enero no. 929, 37000 Leon; ${ }^{3}$ Research Unit UMAE 1, IMSS, \\ Blvd. López Mateos S/N, 37000 Leon, Guanajuato, Mexico
}

Received May 25, 2010; Accepted July 21, 2010

DOI: 10.3892/ijmm_00000515

\begin{abstract}
Global DNA hypomethylation potentially leading to pro-atherogenic gene expression occurs in atherosclerotic lesions. However, limited information is available on the genomic location of hypomethylated sequences. We present a microarray-based survey of the methylation status of $\mathrm{CpG}$ islands (CGIs) in 45 human atherosclerotic arteries and 16 controls. Data from 10,367 CGIs revealed that a subset (151 or $1.4 \%$ ) of these was hypermethylated in control arteries. The vast majority (142 or 94\%) of this CGI subset was found to be unmethylated or partially methylated in atherosclerotic tissue, while only 17 of the normally unmethylated CGIs were hypermethylated in the diseased tissue. The most common functional classes among annotated genes adjacent to or containing differentially methylated CGIs, were transcription (23\%) and signalling factors (16\%). The former included $H O X$ members, PROXI, NOTCH1 and FOXP1, which are known to regulate key steps of atherogenesis. Expression analysis revealed differential expression of all CGI-associated genes analysed. Sequence analysis identified novel DNA motifs with regulatory potential, associated with differentially methylated CGIs. This study is the first large-scale analysis of DNA methylation in atherosclerosis. Our data suggest that aberrant DNA methylation in atherosclerosis affects the
\end{abstract}

Correspondence to: Dr Silvio Zaina, Department of Medical Sciences, Division of Health Sciences, Leon Campus, University of Guanajuato, 20 de Enero no. 929, 37000 Leon, Guanajuato, Mexico E-mail: szaina@leon.ugto.mx

Abbreviations: AA, atherosclerotic artery; CA, control artery; CGI, CpG island; DM-CGI, CpG island that is differentially methylated between atherosclerotic and control arteries; HH-CGI, $\mathrm{CpG}$ island that is hypermethylated in both atherosclerotic and control arteries

Key words: DNA methylation, atherosclerosis, $\mathrm{CpG}$ island transcription of critical regulatory genes for the induction of a pro-atherogenic cellular phenotype.

\section{Introduction}

Atherosclerosis risk is in part determined by dietary and lifestyle-related factors, suggesting that diet- and environment-gene interactions are among the key phenomena in the aetiology and natural history of the disease. Epigenetics offers in principle a molecular model of such interactions, by envisioning that dietary and environmental risk factors modulate gene expression by laying down aberrant DNA methylation and histone post-translational modification patterns, which in turn convert permissive, relaxed chromatin into non-permissive, compact chromatin, or vice versa, depending on the specific gene involved (1). Accordingly, elegant animal models offer proof of principle for the idea that simple dietary interventions can have a significant impact on DNA methylation (2). As for atherosclerosis, potentially predisposing abnormal DNA methylation patterns have been detected in animal models prior to the appearance of vascular lesions (3). Furthermore, human and animal studies consistently indicate that global DNA hypomethylation accompanies advanced atherosclerosis $(3,4)$. Importantly, 2 recent human studies have indicated that circulating levels of inflammation markers and exposure to environmental risk factors for atherosclerosis were associated with hypomethylation of highly repeated elements of leukocyte DNA $(5,6)$. Identifying the sequences undergoing demethylation in atherosclerosis could provide important insights into the molecular mechanisms of atherogenesis and possibly of diet- or environment-gene interactions (7). We therefore conducted an array-based analysis of the DNA methylation status of $\mathrm{CpG}$ islands (CGIs) in control (CAs) and atherosclerotic arteries (AAs). CGIs are discrete sequences present both in intra- and intergenic regions, including the promoters of about half of the human genes (8). The observation that promoter CGI hypermethylation is usually associated with gene silencing in a number of physiological situations including cancer, suggests the pivotal role of CGI DNA methylation in transcription regulation (9). Our study is the first large-scale description of sequences undergoing aberrant DNA methylation in atherosclerosis. 


\section{Materials and methods}

Tissue samples. All relevant procedures were approved by the local ethics committee. Atherosclerotic artery samples were obtained from ischaemic cardiopathy patients undergoing revascularization surgery. Although the atherosclerotic lesions were not histologically classified for severity, our samples consisted of advanced stage lesions, according to surgery recommendations. A fragment of the coronary artery at the lesion site, including the lesion and underlying media, was obtained during the application of the aorta-coronary bridge. The control samples were aortic fragments from patients undergoing aortic valve replacement surgery and with no coronary atherosclerotic lesions, as was indicated by cardiac catheterization and confirmed during surgery. Diabetes was the only exclusion criterion for both groups. Tissue samples were collected in RNAlater (Ambion) and transferred to $-20^{\circ} \mathrm{C}$ within $2 \mathrm{~h}$ from surgery.

Array analysis. Two pools of DNA were prepared, representing equal amounts of the control $(n=16)$ or atherosclerotic $(n=45)$ artery samples, respectively. The 2 groups did not differ in gender (2:1 male:female ratio) or age (22-79 years, $\mathrm{p}>0.05$ ). Hypermethylated DNA fractions were prepared by digestion with the methylation-sensitive enzyme, HpaII, and HpaII-end containing (i.e. unmethylated) fragments were eliminated by exonuclease III treatment according to the method described by Shann et al (10), followed by amplification with the WGA system (Sigma). Reference DNA was prepared by mock digestions in which HpaII was omitted. Primers were eliminated by passage through MinElute columns (Qiagen). Array analysis (labelling, hybridization and raw data generation) was performed at the UHN Microarray Centre, Toronto, Canada. The microarray used (HCGI15K; UHN; www.microarrays.ca) was a singlespotted array containing 14,923 CpG-island clones including the HCGI8.1K set of UHN and a new $6.8 \mathrm{~K}$ set generated by sequence-specific primers targeting potential $\mathrm{CpG}$-island clones that are currently not in any of the UHN collections. BLAT-annotated clones with overlapping sequences $>20 \%$ of either clone were considered as duplicates and excluded. Clones with internal repeat sequences, with no or $>1$ BLAT hits and those mapped to the mitochondrial chromosome (M), were also excluded. Two series of arrays in triplicate, one for the control and the other for the atherosclerotic samples, were co-hybridized with Alexa647-labelled (red) HpaII-digested DNA and Alexa555-labelled (green) reference DNA. The background-subtracted Alexa647 and Alexa555 intensities were normalized to the overall median Alexa555 intensity calculated on all the arrays, and the $\log _{2}$ of the Alexa647/Alexa555 ratio was calculated for each individual array as previously reported (10). Comparisons between the 2 array groups were conducted by ANOVA. Array data have been deposited into the GEO database (www.ncbi.nlm.nih.gov/ projects/geo/) with accession no. GSE15552.

Array data validation. Array data for 10 differentially methylated CGIs were validated by methylation-sensitive PCR (MS-PCR) in 10 more samples from each group with the same characteristics as the ones used for the array analysis. In brief, $10 \mathrm{ng}$ DNA were digested with $1 \mathrm{U}$ McrBC overnight and a 200-300 bp CGI portion was amplified by 35-cycle PCRs. Primers were designed with BiSearch (bisearch.enzim.hu) and their sequences are available upon request (11). Band intensity was quantified with Quantity One software (Bio-Rad) and normalized to the median intensity of the PCR products of the mock-treated DNA (input) made equal to the arbitrary value of 1 . MS-PCRs for each gene were run and analysed on the same gel to reduce variability.

Gene expression analysis. RNA was extracted with the RNeasy system (Qiagen) and cDNA was prepared from $3 \mu \mathrm{g}$ RNA pooled from the same 10 samples used for MS-PCR, using the Access RT-PCR system (Promega). For each gene, one-fiftieth of cDNA was amplified by using the LightCycler FastStart DNA Master ${ }^{\text {PLUS }}$ SYBR Green I (Roche) according to the manufacturer's instructions in a LightCycler 1.5 machine (Roche), with primers designed in qPrimerDepot (primerdepot.nci.nih.gov). Primer sequences are available upon request. $\mathrm{Ct}$ values were calculated using a fluorescence value equal to twice the baseline as the threshold. The expression was normalized by calculating the ratio between the $\mathrm{Ct}$ of a sample and the average $\mathrm{Ct}$ for $G A P D H$ in the same group CAs or AAs.

Bioinformatics. For bioinformatics analysis, CGI annotations were as provided by the array supplier and were based on NCBI UniGene human build 217 and UCSC human build hg18. Genomic localization was determined with the NCBI Map Viewer resource (www.ncbi.nlm.nih.gov). CG content and $\mathrm{CpG}$ density ( $\mathrm{CpG}$ count/CGI length ratio) were determined with the EMBOSS CG and $\mathrm{CpG}$ report tools (oz.ifc.unam.mx/EMBOSS). Gene functions were deduced according to the categories on the Gene Ontology database (www.geneontology.org). DNA motif search was conducted with MEME software (meme.sdsc.edu) using default parameters (12). DNA motifs were compared against the databases of known protein-binding DNA sequences, namely the TRANSFAC and JASPAR core databases, using TOMTOM (meme.nbcr.net/meme4_1/cgi-bin/tomtom.cgi).

\section{Results}

CGI methylation status in CAs and AAs. Our array-based survey yielded reliable data on the methylation status for 10,367 CGIs. We classified CGIs as unmethylated if the Alexa647/Alexa555 ratio was $<0.3$, or if $\log _{2}$ Alexa647/ Alexa555 $\left(\log _{2} \mathrm{r} / \mathrm{g}\right)$ was $<-1.6$, representing an excess hybridized reference DNA (i.e. not HpaII digested) compared to filtered DNA. CGIs were considered to be hypermethylated if the Alexa647/Alexa555 ratio was $>3$, corresponding to $\log _{2} \mathrm{r} / \mathrm{g}>1.6$. Intermediate values were considered as partial hypo- or hypermethylation (Fig. 1A).

In accordance with the well established notion that the vast majority of CGIs are unmethylated (8), most of the $(9,085$ or $87.6 \%)$ CA CGIs fell within the unmethylated class and accordingly, the overall median CA CGI $\log _{2} \mathrm{r} / \mathrm{g}$ was -2.4 . In contrast, the hypermethylated CGIs were a minority (151 


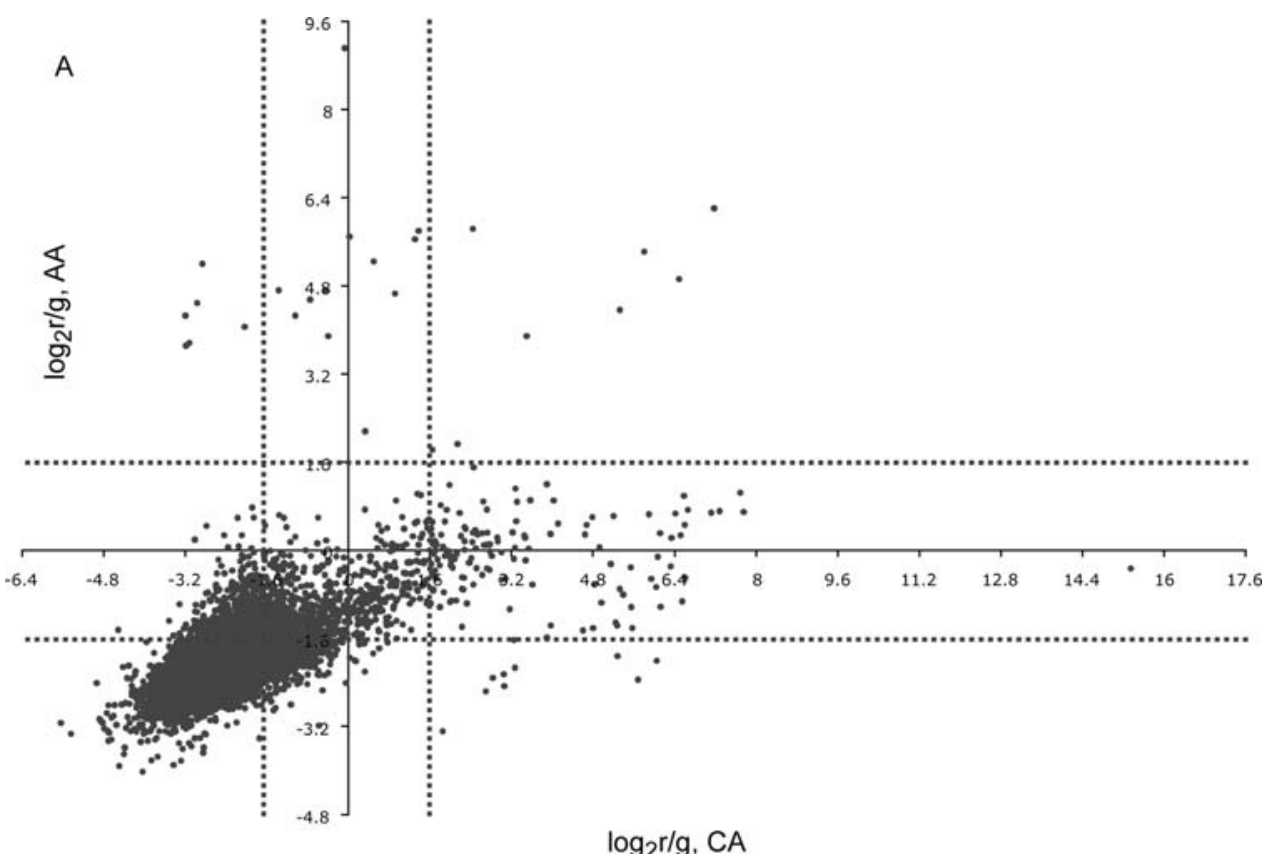

$\log _{2} r / g, C A$

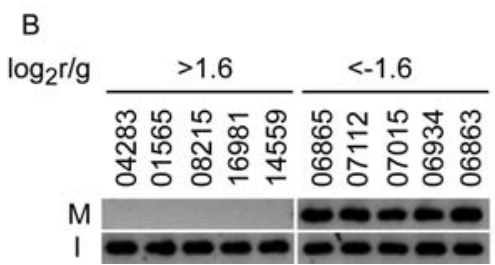

Figure 1. Global pattern of CGI methylation. (A) Plot of CGI $\log _{2} \mathrm{r} / \mathrm{g}$ values in CAs and AAs. Each data point represents the methylation status of an individual CGI in CAs and AAs. Vertical and horizontal dashed lines represent the $\log _{2} \mathrm{r} / \mathrm{g}$ values of -1.6 and 1.6 on each axis and therefore separate hypermethylated, unmethylated and intermediately methylated CGIs in either group. (B) Validation of array-deduced methylation status of 5 hypermethylated and 5 unmethylated CGIs (left and right panels, respectively) in CAs by McrBC digestion-based MS-PCR. CGIs are referred to using the last 5 digits of the UHN clone nomenclature, i.e. UHNhscpg00XXXXX. M and I, McrBC-digested and input (undigested) DNA, respectively.

or $1.4 \%$ ) in this group. In order to corroborate the array results, the methylation status of 10 randomly chosen un- or hypermethylated CA CGIs was verified by MS-PCR in the DNA pool used for the hybridizations (Fig. 1B).

The comparison of the CGI methylation status between CAs and AAs revealed significant $(\mathrm{p}<0.01)$ changes. Of the 151 normally hypermethylated CGIs, 142 (or 94\%) were found to be unmethylated or in an intermediate methylation status in AAs (Fig. 1A). Conversely, only 17 of the CGIs that were unmethylated or intermediately methylated in CAs, were hypermethylated in AAs. We validated the methylation status of 10 CGIs differentially methylated between AAs and CAs (DM-CGIs) adjacent to relevant genes by MS-PCR in 10 more age- and gender-paired samples not included in the array analysis (Fig. 2A). Validation examples of DM-CGI methylation status are shown in Fig. 2B. Further characterization was carried out of those differentially methylated CGIs that showed the most extreme changes in DNA methylation, i.e. were hypermethylated in one group $\left(\log _{2} \mathrm{r} / \mathrm{g}>1.6\right)$ and incompletely methylated or unmethylated (i.e. showing an Alexa647/Alexa555 ratio $<0.75$, corresponding to $\left.\log _{2} \mathrm{r} / \mathrm{g}<-0.4\right)$ in the other group, with a p-value of $<0.01$. Excluding BLAT-unmatched clones,
45 CGIs hypermethylated in CAs and 10 in AAs met these criteria. These CGIs will be referred to as DM-CGIs.

Genomic location of DM-CGIs. A recent study of normal, non-vascular human tissues has shown that CGIs with tissuespecific methylation status are found in specific genomic locations, thus providing insights on the mechanisms and transcriptional impact of differential methylation (13). In order to assess whether comparable patterns occur in atherosclerosis, we analysed the genomic locations of DM-CGIs $(n=45)$ and non-DM-CGIs that were hypermethylated in both CAs and AAs (HH-CGIs; $n=8$ ). Twenty-nine DM-CGIs were intragenic and 26 were intergenic, whereas the majority of HH-CGIs (6 out of 8) was intragenic. These CGIs differed in their distribution (Fig. 3). DM-CGIs unmethylated in AAs tended to be localized preferentially in promoters (i.e. 5' UTR, TSS and $1.0 \mathrm{~kb}$ upstream to the latter) and first introns. In contrast, DM-CGIs hypermethylated in AAs showed a broader distribution encompassing $5^{\prime}$ and middle third gene portions $(p<0.03$ in comparison with the latter group) Intragenic HH-CGIs, however, were preferentially positioned at middle third gene portions $(\mathrm{p}<0.02$ in comparison with either DM-CGI class distribution). All 3 CGI classes were 
A

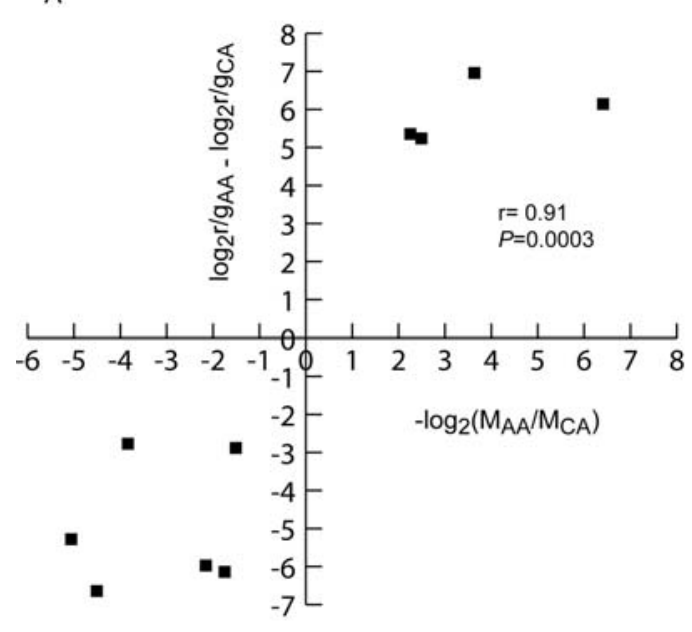

B

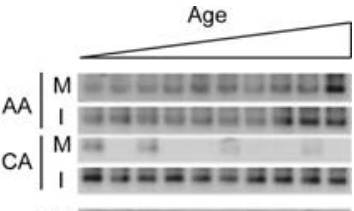

19681

AA $\begin{gathered}\mathrm{M} \\ \mathrm{I} \\ \mathrm{CA} \\ \mathrm{M} \\ \mathrm{I}\end{gathered}$

26224

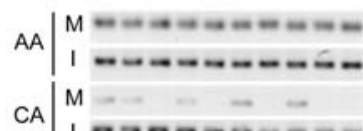

24686

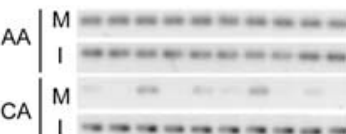

24694

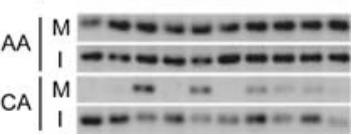

Figure 2. Validation of selected DM-CGI methylation status. (A) Correlation between MS-PCR data (X-axis) and array data (Y-axis) for 10 validated DM-CGIs. $\mathrm{M}_{\mathrm{CA}}$ and $\mathrm{M}_{\mathrm{AA}}$ are average normalized MS-PCR band intensities in CAs and AAs, respectively. The Alexa647/Alexa555 ratios in CAs and AAs are r/g $\mathrm{g}_{\mathrm{CA}}$ and $\mathrm{r} / \mathrm{g}_{\mathrm{AA}}$, respectively. Negative and positive values indicate hypo- and hypermethylation in AAs relative to CAs, respectively. (B) MS-PCR analysis of 5 representative CGIs in 10 age- and gender-paired samples. Age difference between the paired samples was $<5$ years. Numbers on the left are the last 5 digits of the CGI clone notation of UHN, i.e. UHNhscpg00XXXXX. M and I, McrBC-digested and input (undigested) DNA, respectively. Absent or decreased bands indicate a relative hypermethylation.

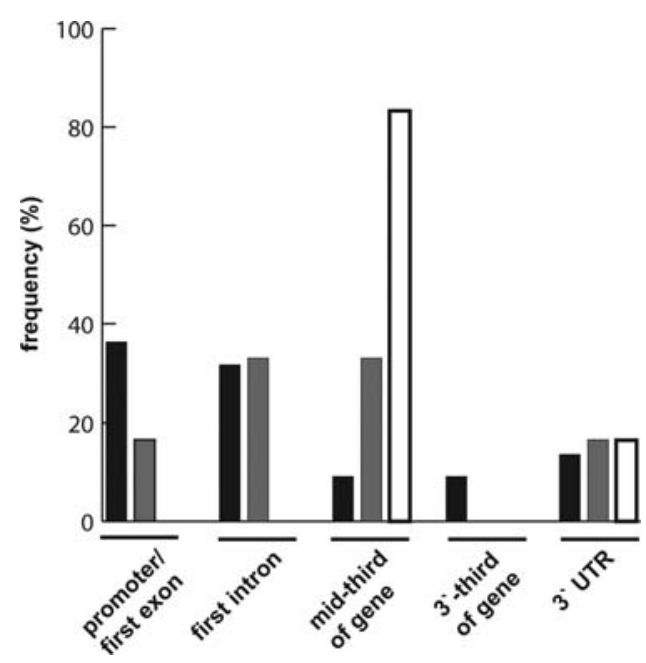

Figure 3. Distribution of intragenic DM-CGIs and HH-CGIs across different gene portions. Black, DM-CGIs unmethylated in AAs; grey, DM-CGIs hypermethylated in AAs; white, HH-CGIs.

similarly represented at 3' UTR regions (Fig. 3). Of the 20 DMCGIs and HH-CGIs that were not within promoters or $3^{\prime}$ UTR regions, the vast majority (17 or $85 \%$ ) was located in introns rather than in protein coding sequences.

As for intergenic DM-CGIs, their distance from flanking genes varied (i.e. 1.2-120 kb). Of this CGI group, 14 (or $54 \%$ ) were the only array-represented CGIs positioned between the respective up- or downstream genes, while 11 were the closest CGIs to either the respective down- or upstream gene. Furthermore, DM-CGIs that were unmethylated in AAs were in average $~ 100$-fold more distant from downstream or upstream genes than DM-CGIs hypermethylated in
AAs or intergenic HH-CGIs $(350.5+678.1,2.6+2.4$ and $2.7+2.2 \mathrm{~kb}$, respectively; $\mathrm{p}<0.001)$.

Identity and expression of DM-CGI-associated genes. The functional classification of annotated genes upstream or downstream to, or containing DM-CGIs, revealed several gene functions, with a bias towards transcription factors (TFs), representing the most numerous class, followed by signalling factors and genes involved in organelle homeostasis (Table I). A description of the DM-CGI genomic location and adjacent annotated genes is presented in Table II. Furthermore, we analysed the expression of TFs and atherosclerosis-related genes adjacent to, or containing the $10 \mathrm{DM}$ CGIs for which we validated the methylation status, by quantitative RT-PCR in the same 10 samples used for MSPCR (Fig. 4, graphs on the right). A schematic representation of the regions containing validated DM-CGIs is shown in Fig. 4, left side. Differential DNA methylation coincided with changes in expression in all the cases analyzed $(p<0.03$ in comparison with normalized $\mathrm{Ct}$ ). The methylation status of several DM-CGIs was inversely related with gene expression. Two intergenic DM-CGIs located in the HOX TF clusters were one notable example. One DM-CGI was located downstream to HOXD10 between HOXD4 and HOXD8 in chromosome 2 and was hypermethylated in AAs, while the other was located in a region of chromosome 12 including HOXC9, HOXClO and HOXC11, and was unmethylated in AAs. The expression of HOXC9, HOXC10 and HOXD10 was inversely related to the methylation status of the associated DM-CGI (Fig. 4A and B). The same pattern was observed in a DM-CGI unmethylated in AAs occupying the EGFL7 promoter and downstream to the TF gene, NOTCH1 (Fig. 4C and D). Two other DM-CGIs lay $\sim 1 \mathrm{Mb}$ apart in a region of chromosome 1 in the vasohibin 2 (VASH2) promoter and 
Table I. Functional classification of genes upstream or downstream to, or containing DM-CGIs, ordered by class size, with the percentage $(\%)$ of total gene count in each class. ${ }^{a}$

\begin{tabular}{|c|c|c|c|}
\hline \multirow[t]{2}{*}{ Gene function } & \multicolumn{2}{|c|}{ DM-CGI methylation status in AAs } & \multirow[t]{2}{*}{$\%$} \\
\hline & Hypermethylated & Unmethylated & \\
\hline Transcription & $\begin{array}{l}\text { HOXD } 4, \text { HOXD } 8 \\
\text { ASCL1, PROX1 }\end{array}$ & $\begin{array}{l}\text { LHX1, DMRTA2, RUNX3, SMAD2, ZC } 3 H 13 \\
\text { ZNF184, ZNF253, ZNF506, ZNF821, NOTCH1, } \\
\text { CITED2, ZNHIT2, DIP2A, ZFP1, GSC, } \\
\text { MORF4L2, HOXC11, HOXC10, FOXP1 }\end{array}$ & 23.0 \\
\hline Signalling & $F K B P 1 A, R P S 6 K C 1$ & $\begin{array}{l}\text { CENTB5, GPR143 GPR20, GNB1L, ASZ1 } \\
\text { ESRRG, S10OB, CAMK1D, TAOK1, MGC13024 } \\
\text { GAL, ASB3, EGFL7 }\end{array}$ & 15.6 \\
\hline Organelles & NSFL1C & $\begin{array}{l}\text { GORASP1, WDR 48, AP1G1, MUTED } \\
\text { KIAAO174, IER3IP1, M6PRBP1 }\end{array}$ & 8.3 \\
\hline Protein catabolism & $U B E 2 D 4$ & FAU, ZNRF1, SERPINA13, HERC6 & 5.2 \\
\hline Transport & & CLIC4, APXL, SLC45A4, KCNJ1, CFTR & 5.2 \\
\hline Apoptosis & & FAF1, SYVN1, MCPH1, P53AIP1 & 4.2 \\
\hline Cell adhesion & $C D H 4, D C C$ & $A S A M, C A D M 1, N R X N 1$ & 4.2 \\
\hline Chromatin & $M B D 2$ & HIST1H2BL, TBL1X, SETD8 & 4.2 \\
\hline Lipid metabolism & & ACBD5, PIGY, PIB5PA, AGPAT2 & 4.2 \\
\hline Cytokine & CTF1 & $L I F, O S M$ & 3.1 \\
\hline Cytoskeleton & & MYOM2, SMTN, CAPZB & 3.1 \\
\hline Mitochondrial ribosome & MRPS24 & MRPL48, MRPS9 & 3.1 \\
\hline Non-lipid metabolism & $P A H$ & PUSL1, PRMT2 & 3.1 \\
\hline Cell proliferation & $I G F 1$ & $A P C$ & 2.1 \\
\hline Inflammation & & NAGA, TICAMI & 2.1 \\
\hline Redox & FLJ39599 & TXNRD & 2.1 \\
\hline Testis-specific & & HORMAD2, DNAJB13 & 2.1 \\
\hline Angiogenesis & VASH2 & & 1.0 \\
\hline Erythropoiesis & $F L V C R$ & & 1.0 \\
\hline Hypoxia & & $L D H D$ & 1.0 \\
\hline Micro-RNA & & DICER1 & 1.0 \\
\hline
\end{tabular}

aIf multiple functions have been reported for a gene, the function relevant to atherogenesis is listed.

upstream to PROX1, another member of homeobox TFs, respectively (Fig. 4E and F). Hypermethylation of DM-CGIs in AAs coincided with transcriptional down-regulation of the latter 2 genes. Similarly, hypomethylation of a 3' UTR DMCGI coincided with up-regulation of the $S 100 B$ gene and promoter hypermethylation was associated with $M B D 2$ down-regulation in AAs (Fig. 4G and $\mathrm{H}$ ). In contrast with the above patterns, hypomethylation of 2 intragenic DM-CGIs was associated with expression down-regulation in the case of smoothelin (SMTN) and TF FOXP1 (Fig. 4I and J).

Furthermore, we confirmed the methylation status of 3 DM-CGIs previously reported to have a hypermethylation tendency in atherosclerosis (i.e. in the 5' region of ESR2, FADS2 and TFPI2) (14-16). $\log _{2} \mathrm{r} / \mathrm{g}$ values in CAs and AAs were $-2.72,-1.40$ for $E S R 2$, and $-3.14,-2.18$ for $F A D S 2$, respectively, with a p-value of $<0.05$ in both cases. Corresponding values for TFPI2 were -2.27 and -2.11 with no significant difference, and were in line with the observation that hypermethylation at that gene was present in a minority of lesions (16). Other promoters previously studied in atherosclerosis were not represented in the arrays used here.

Bioinformatic analysis of DM-CGI. We searched for sequence features in DM-CGIs and HH-CGIs that could yield further insights into the mechanisms of CGI differential methylation. The comparison of CGI size, CG content and $\mathrm{CpG}$ density (no. of $\mathrm{CpG}$ to CGI length ratio), a parameter shown to be related with CGI methylation status in somatic cells (17), did not reveal any significant difference. Furthermore, we screened for DNA motifs unique for any CGI class in $4 \mathrm{~kb}$-sequences centered at the CGI midpoint. The presence of class-specific DNA motifs could signal the possible involvement of DNA-binding factors in the establishment of differential DNA methylation. The MEME software yielded a 29-bp motif (DMM) that was present in $55.5 \%$ of the DMCGIs unmethylated in AAs $(\mathrm{E}=2.2 \mathrm{e}-118)$ but absent in the DM-CGIs hypermethylated in AAs or the HH-CGIs (Fig. 5A). The lack of DMM detection in HH-CGIs was not due to 
Table II. DM-CGIs with genomic location, associated genes and average $\log _{2} \mathrm{r} / \mathrm{g}$ values in CAs and AAs.

\begin{tabular}{|c|c|c|c|}
\hline CGI clone & Genomic location & Within & Upstream \\
\hline
\end{tabular}

DM-CGIs hypermethylated in CAs, unmethylated in AAs

UHNhscpg0000083 chr17:77921639-77922214

UHNhscpg0000313

UHNhscpg0001565

UHNhscpg0002002

UHNhscpg0003364

UHNhscpg0005293

UHNhscpg0005702

UHNhscpg0005813

UHNhscpg0006769

UHNhscpg0008215

UHNhscpg0008474

UHNhscpg0009592

UHNhscpg0013290

UHNhscpg0014151

UHNhscpg0014542

UHNhscpg0014599

UHNhscpg0014796

UHNhscpg0014912

UHNhscpg0015048

UHNhscpg0015225

UHNhscpg0015321

UHNhscpg0015763

UHNhscpg0016316

UHNhscpg0017732

UHNhscpg0017800

UHNhscpg0018821

UHNhscpg0018976

UHNhscpg0019872

UHNhscpg0020507

UHNhscpg0020592

UHNhscpg0022803

UHNhscpg0022884

UHNhscpg0023790

UHNhscpg0023931

UHNhscpg0024686

UHNhscpg0024689

UHNhscpg0024694

UHNhscpg0024745

UHNhscpg0025462

UHNhscpg0025899

UHNhscpg0026224

UHNhscpg0027388

UHNhscpg0027650

UHNhscpg0027678

UHNhscpg0028047

UHNhscpg0028140
UHNhscpg0027684 chr2:51112865-51113731

chr3:39123016-39123538

chr12:52663262-52664017

chr14:94304731-94304947

chr1:25055302-25055491

chr12:122434216-122434426

chr13:45322805-45324127

chr1:1230036-1231291

chr3:71197019-71197553

chr11:128280580-128280993

chr18:43041077-43041115

chr7:116906424-116907180

chr2:104835710-104836266

chr11:115092453-115092670

chr10:27570656-27571247

chr6:140018073-140018373

chr11:122734054-122734895

chr19:4780161-4780685

chr1:215376726-215377012

chr17:24645319-24645686

No BLAT hit

No BLAT hit

chrX:102829282-102829630

chr1:19635271-19635541

chr19:19793020-19793758

chr6:27678451-27678629

chr11:64645232-64646526

chr8:142314675-142315035

chrX:9693151-9694306

chr11:73264949-73265794

chr11:68193727-68194620

chr16:73705572-73706745

chr17:32239295-32240467

chr21:46842417-46843516

chr22:18221859-18223031

chr22:29824296-29825316

chr22:40799574-40800700

chr6:8008992-8009814

chr8:2062660-2063586

chr9:138679621-138680856

chr4:89597065-89598125

chr10:12430778-12432679

chr5:111782224-111784173

chr22:28971571-28973279

chr16:70451013-70451959

chr1:50665901-50666652

$\begin{array}{lll} & \text { FLJ35767 } & \text { FLJ2222 } \\ \text { GORASP1 } & \text { NRXN1 } & \text { ASB3 } \\ & \text { HOXC11 } & \text { HOXC10 } \\ \text { GSC } & \text { SERPINA13 } & \text { DICER1 } \\ & \text { CLIC4 } & \text { RUNX3 } \\ & \text { SBNO1 } & \text { SETD8 } \\ \text { FOXP1 } & \text { NURIT } & \text { KIAA0853 } \\ \text { C11 } 0 \text { Ff45 } & \text { EENTB5 } & \text { PUSL1 } \\ & \text { KCNJ1 } & \text { LOC100289131 } \\ & \text { IER3IP1 } & \text { P53AIP1 } \\ & \text { ASZ1 } & \text { CFTR } \\ & \text { CR936735 } & \text { MRPS9 } \\ & \text { BC047021 } & \text { MGC13125 } \\ & \text { ACBD5 } & \text { LOC387646 } \\ & \text { CITED2 } & \text { AK097143 } \\ & \text { ASAM } & \text { AK127457 } \\ & \text { TICAM1 } & \text { M6PRBP1 } \\ & \text { ESRRG } & \text { GPATC2 } \\ & \text { NUFIP2 } & \text { TAOK1 } \\ & & \end{array}$

$\begin{array}{lll} & \text { MORF4L2 } & \text { TMEM31 } \\ \text { CAPZB } & \text { AK096102 } & \text { C1orf151 } \\ \text { ZNF506 } & \text { FLJ46230 } & \text { ZNF253 }\end{array}$

FKSG56 ZNF184 HIST1H2BL

FAU

GPR143

CHCHD8 MRPL48

ZNHIT2

SLC45A4

TBL1X

SAPS3

LDHD

ZNRF1

MRM1

S100B

GNB1L

DIP2A

SMTN BC012026

FAM109B

MUTED

MYOM2

EGFL7

HERC5

CAMK1D

LIF

LOC55565

\section{NAGA}

BC028580

KBTBD11

NOTCH1

HERC6

C10orf7

EPB41L4A

HORMAD2

AP1G1

AJ301580

DM-CGIs unmethylated in CAs, hypermethylated in AAs

UHNhscpg0001071

UHNhscpg0003554

UHNhscpg0005354

UHNhscpg0011135 chr1:212222309-212223400

chr20:1320740-1321479

chr7:43912050-43912251

chr1:211190078-211190296
FKBP1A

URG4

VASH2
RPS6KC1

AK000809

MRPS24

FLVCR
FAF1

PROX1

NSFL1C

UBE2D4

VASH2
2.29070985

2.226160184

6.521206416

1.994891121

6.091264337

6.016275549

1.768802278

2.97263872

3.237808178

5.359131933

1.69480582

3.580301757

2.195421389

2.590023363

2.078305966

5.520081361

2.416687107

5.242359626

3.047119619

2.199685837

5.909880104

1.642190065

2.235284245

1.634887846

4.799718052

4.571042478

3.214585772

5.544692553

4.766596074

3.858298486

5.651905748

1.961095511

5.211070651

2.66772773

3.940243041

1.632872597

5.30028017

5.252898787

6.572617804

4.932429293

6.004100257

1.846248553

3.134990152

3.015604131

3.022969021

2.200354593

2.80695587

$-0.935517059$

$-0.681858958$

$-0.903185685$

$-0.886415227$

$-0.998422156$

$-1.983046692$

$-1.000565333$

$-0.505776329$

$-2.101301822$

$-0.778913464$

$-0.486156485$

$-0.595334021$

$-0.460685003$

$-0.42021637$

$-0.887925062$

$-1.006617213$

$-0.59569034$

$-1.341504446$

$-0.492737606$

$-1.36364171$

$-0.493664785$

$-0.684376507$

$-0.470463224$

$-0.703610948$

$-0.589762553$

$-1.42677703$

$-1.601708402$

$-1.381365105$

$-1.382565412$

$-1.553981206$

$-2.321222231$

$-0.909580258$

$-1.284382721$

$-2.534658496$

$-1.339843615$

$-0.423449001$

$-0.671827614$

$-1.895368556$

$-0.471029058$

$-0.926405025$

$-0.637057408$

$-0.73415746$

$-1.040169498$

$-2.219980056$

$-2.441966395$

$-0.673368865$

$-2.283181688$

$-1.068343579$

4.284862484

3.790954386

3.918773175

4.75654165 
Table II. Continued.

\begin{tabular}{|c|c|c|c|c|c|c|}
\hline CGI clone & Genomic location & Within & Upstream & Downstream & $\begin{array}{c}\text { Average } \\
\log _{2} r / g C A\end{array}$ & $\begin{array}{c}\text { Average } \\
\log _{2} \mathrm{r} / \mathrm{g} \text { AA }\end{array}$ \\
\hline UHNhscpg0014640 & $\operatorname{chr} 16: 15435454-15435917$ & C16orf45 & FLJ39599 & LKAP & -0.780517895 & 4.578974225 \\
\hline UHNhscpg0015177 & chr16:30840387-30840828 & LOC283932 & CTF1 & MGC13024 & -3.222082358 & 4.287964286 \\
\hline UHNhscpg0016809 & chr18:49929963-49930163 & & DCC & MBD2 & -1.392033622 & 4.748487633 \\
\hline UHNhscpg0019681 & chr2:176723074-176723546 & & HOXD9 & HOXD4 & -3.214739959 & 3.743257903 \\
\hline UHNhscpg0024537 & chr20:59552448-59553578 & $\mathrm{CDH} 4$ & LOC284757 & $\mathrm{CDH} 4$ & -2.064500246 & 4.083960366 \\
\hline UHNhscpg0028067 & $\operatorname{chr} 12: 101834908-101835700$ & PAH & IGF1 & ASCL1 & -2.895150067 & 5.229689851 \\
\hline
\end{tabular}

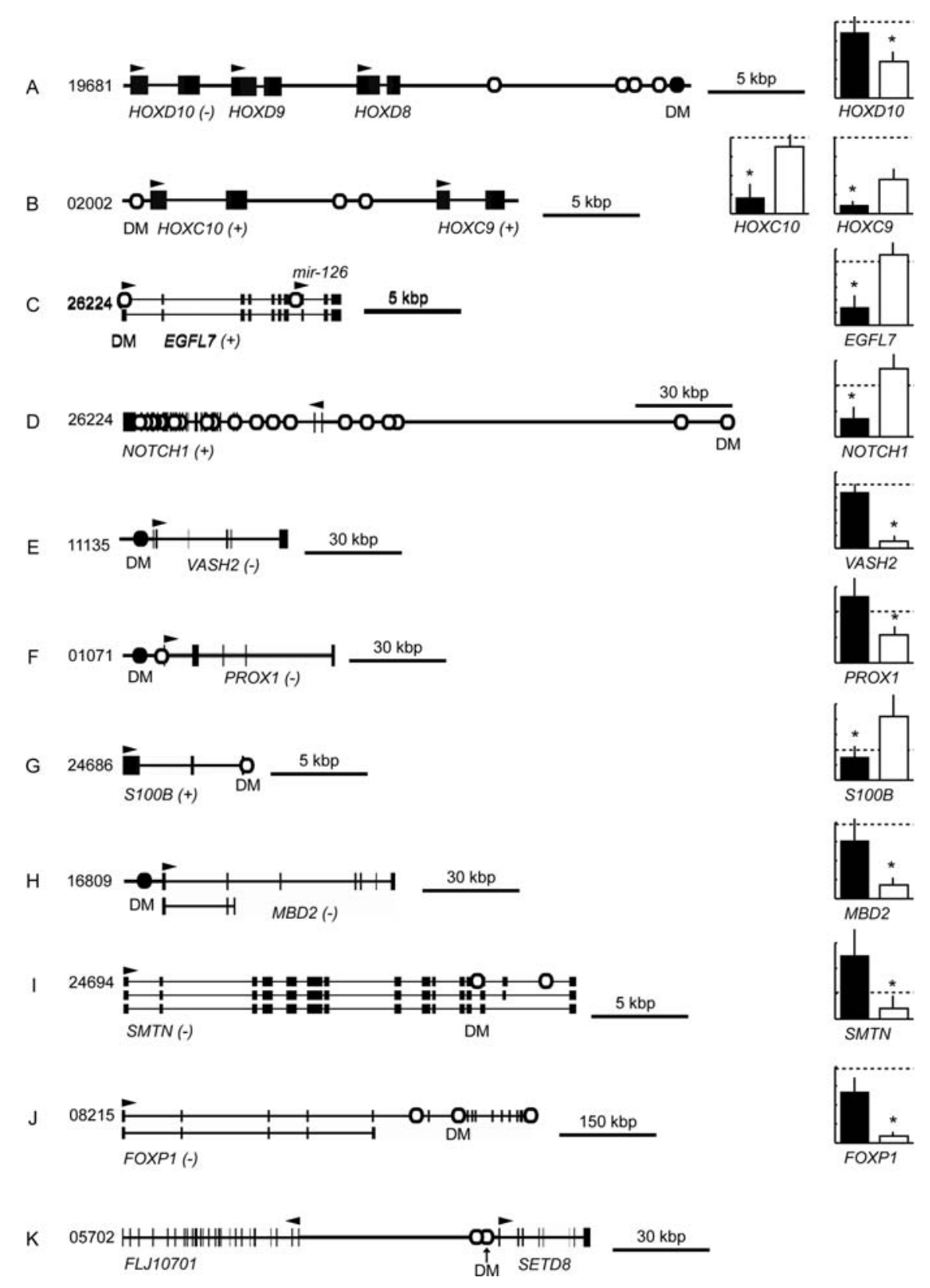

Figure 4. Schematic map of 10 validated DM-CGI-containing regions (left) and the expression of the relevant annotated genes (right). In the diagrams on the left, open and solid ovals are unmethylated and hypermethylated CGIs, respectively. CGIs that are DM-CGIs (indicated as DM) are marked by an arrow and their methylation status in AAs is shown. (+) or (-) beside the gene names indicates up- and down-regulation in AAs, respectively. In the case of unlabelled genes, expression analysis was not carried out. The arrowhead above the genes indicates the start site and the direction of transcription. CGIs are referred to using the notation of UHN as indicated in the legend of Fig. 1. Eleven sequences are shown as the position of CGI 26224 is shown both relative to NOTCHI and EGFL7. Right graphs, quantitative RT-PCR expression analysis of genes indicated below each graph. Mean and SD of normalized expression are shown. Black and white bars, CAs and AAs, respectively. For simplicity, numbers are not shown in the value axis, but the normalized expression value=1 is indicated as reference by the horizontal dashed lines. " $\mathrm{p}<0.05$ in comparison between the CAs and AAs. 

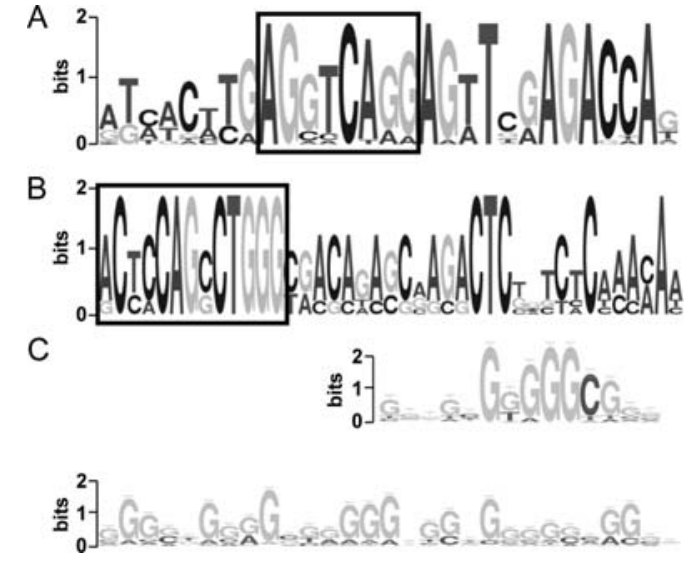

Figure 5. DNA motifs specific for either DM- or non-DM-CGIs. Motifs are represented as sequence logos produced by the MEME software. The height of a letter in a stack indicates the probability of the letter to be represented at that position. (A and B) DMM and non-DMM, respectively. Boxes contain motif portions that are conserved in the M. musculus. (C) Krox binding sitehomologous motif. The sequence in the lower part of panel $\mathrm{C}$ is the motif query, the upper sequence indicates the Krox motif (accession no. M00982).

sample size, as repeated analyses of randomly chosen groups of $n=8$ DM-CGIs always identified the motif. Neither the DMM presence nor copy number correlated with DM-CGI size, but the DMM copy number was weakly correlated with $\mathrm{CpG}$ density $(\mathrm{r}=0.42, \mathrm{p}<0.02)$. DMM was not preferentially associated with intra- or intergenic DM-CGIs, but the DMMassociated DM-CGIs were on average $\sim 10$-fold closer to downstream genes than non-DMM-associated DM-CGIs $(56.8 \pm 50.0$ and $644.2 \pm 511.1 \mathrm{~kb}$, respectively, $\mathrm{p}<0.03)$. Furthermore, we detected a candidate non-DM-CGI motif (non-DMM) present in 6 out of the $8 \mathrm{HH}-\mathrm{CGIs}$ but not present in any of the DM-CGIs (E=3.3e-14; Fig. 5B). Both DMM and non-DMM were invariably located outside CGIs. The analysis of mouse CGIs with BLAST homology with DMMassociated DM-CGI revealed a conserved 5'-AGNNCAGG-3' sequence present in DMM, and a 5'-ACTCCAGNCTGGG-3' sequence present in non-DMM (Fig. 5A and B). Neither DMM nor non-DMM match any known binding site included in the TRANSFAC and JASPAR core databases. In addition, the MEME software yielded a 29-bp DNA motif present in $100 \%$ of the DM-CGIs hypermethylated in AAs and in 12 (or $26.6 \%$ ) of the DM-CGIs unmethylated in AAs. This DNA motif shows homology with the Krox binding site according to the TRANSFAC database ( $\mathrm{p}=8.6 \mathrm{e}-07$; Fig. 5C). The 2 DM-CGI-specific DNA motifs were not mutually exclusive, as 4 DMM-associated DM-CGIs also contained a putative Krox binding site. We also detected CT- and A-rich motifs previously reported in normally hypermethylated CGIs but these were not significantly over-represented in any CGI class analysed here (18).

\section{Discussion}

By examining the DNA methylation status of $\sim 10,000$ CGIs in CAs and AAs, we hereby presented an initial largescale analysis of the epigenetic changes associated with cardiovascular disease. The analysis of CA CGI methylation showed that a small portion of autosomal, not imprinted gene- associated CGIs is hypermethylated, thus supporting previous suggestions that this DNA methylation pattern could be a general feature of somatic tissues $(18,19)$. The vast majority of these normally hypermethylated CGIs is found unmethylated in AAs, indicating that this net CGI demethylation contributes to the global DNA hypomethylation observed in atherosclerotic tissues in previous studies (20).

DM-CGIs were located within or relatively close to a number of genes known to be involved in atherogenesis. Approximately $40 \%$ of the DM-CGI-associated genes were regulatory (i.e. TFs and signalling factors), in accordance with the idea that epigenetic changes are upstream events in the establishment of an atherosclerosis-specific gene expression pattern. In general, CGI differential methylation was associated with up- or down-regulation of genes implicated in angiogenesis, vascular smooth muscle cells (VSMCs) phenotype modulation and inflammation. One remarkable example was the opposite effects on $\mathrm{HOXC}$ and HOXD members, known to promote vascular remodelling and inhibit adult angiogenesis, respectively (22). HOXCs located in physical proximity to a DM-CGI unmethylated in AAs were selectively expressed in AAs, whereas the precisely opposite pattern was observed in $H O X D 10$. A recent study reported that aberrant homeobox CGI methylation is a frequent event in breast cancer, thus suggesting a partially overlapping epigenetic dysregulation of cell proliferation and migration in distinct diseases (23). Furthermore, we observed activation of NOTCH1 and EGFL7, 2 inducers of angiogenesis that are physically associated with the same DM-CGI, and down-regulation of $\mathrm{VASH} 2$, an inhibitor of adult angiogenesis, in AAs (24-26). It has also been reported that demethylation of the EGFL7 promoter activates $m i R-126$, a pro-angiogenic micro-RNA transcribed from an EGFL7 intron (27). Another landmark of atherogenesis is the transition of VSMC from a contractile, differentiated phenotype to a secretory, relatively immature one. This phenomenon is accompanied by a significant re-organization of organelles and secretory vesicles (28). Accordingly, we observed downregulation of the differentiated VSMC marker, SMTN, in AAs, and organelle homeostasis and secretory genes were found associated with DM-CGIs (29). As for inflammation, we observed down-regulation of FOXP1, a forkhead family TF shown to inhibit macrophage activation, and up-regulation of the AGE receptor ligand, S100B $(30,31)$. Relatively few genes involved in chromatin structure regulation were associated with DM-CGIs and their role in atherogenesis is poorly understood. One exception is $M B D 2$, representing a methylcytosine-binding protein that can be induced in cultured VSMCs by stimulation with homo-cysteine, an amino acid implicated in atherogenesis (32).

The analysis of DM-CGI genomic location provided insights into the potential mechanisms of transcriptional control by CGI methylation. In the case of the 10 validated DM-CGIs, the methylation status of the intergenic or promoter DM-CGIs was inversely related with gene expression in accordance with the generally accepted role of DNA methylation in transcription regulation. In contrast, the opposite was observed between methylation and transcription for the intragenic DM-CGI-associated genes, FOXP1 and SMTN. This observation suggests that intragenic DNA methylation 
has a complex impact on gene expression as hinted at by previous studies on plants (33). For all the validated intergenic DM-CGIs, differential CGI methylation was associated with changes in the expression of genes located as far as $120 \mathrm{~kb}$ and separated by up to 5 non-DM-CGIs from the relevant DM-CGI. This observation is reminiscent of the results of a previous study showing that regions of tissuespecific methylation are often distant from genes (34). Thus, our data suggest the testable hypothesis that at least some DMCGIs could be important long-range epigenetic expression regulators.

Our data indicate that DM-CGIs and HH-CGIs show different intragenic distributions. DM-CGIs unmethylated in AAs and HH-CGIs are significantly more abundant in promoters and in the middle third introns of genes, respectively. These results differ from the ones of a recent largescale bioinformatics-based study including a wide range of non-vascular normal human tissues (13). The authors showed that DM-CGIs are significantly more abundant in introns than in other gene compartments, while promoters preferentially include constitutively unmethylated CGIs. In the light of this data comparison, it is conceivable that during atherogenesis, the normal equilibrium between mechanisms that maintain selected (i.e. promoter-associated) CGIs in a constitutively unmethylated state, and other mechanisms establishing tissue-specific CGI hypermethylation is lost in favour of the former. Another difference between our studies is the lack of a substantial association of any DM-CGI or HH-CGI with protein coding regions in our samples. Further study is required to assess whether these discrepancies reflect distinct CGI methylation regulations in normal and diseased cells. Furthermore, our data do not show any significant preference for the inter- or intragenic location of DM-CGIs, whereas the study mentioned above indicates that DM-CGIs are mainly intragenic. Nevertheless, this likely reflects a bias against intergenic elements in the latter CGI collection (13).

DM-CGI nucleotide composition analysis provided limited hints on the mechanisms of differential CGI methylation between AAs and CAs. CpG density did not correlate with the DM- or HH-CGI status, indicating that CGI demethylation in CAs is mechanistically distinct from the preferential demethylation of CpG-dense CGIs in somatic cells $(13,17)$. The presence of CGI class-specific DNA motifs suggests that differential CGI methylation could be regulated by DNAbinding factor(s). As for the yet uncharacterized DMM motif, our data on the distance between DM-CGIs and adjacent annotated genes suggest that this DNA motif could regulate the DNA methylation of CGIs involved in relatively shortrange interactions with downstream genes. The functional significance of the over-representation of the DMM motif in high CpG density DM-CGIs is difficult to interpret, although it could be related to the preferential somatic demethylation of CpG-dense CGIs as mentioned above $(13,17)$. The presence of putative Krox binding sites in a subset of DM-CGIs was expected, since early growth response TFs play a pivotal role in atherogenesis (35). Nevertheless, Krox binding site-associated CGIs showed inconsistent DNA methylation changes between CAs and AAs, making it difficult to assign a straightforward epigenetic function to this motif.
One limitation of the present study was the cell type heterogeneity of AAs compared to CAs, which raises the possibility that at least some of the observed DM-CGI methylation and gene expression patterns were not established de novo in atherosclerosis but were pre-existent in nonvascular cells recruited to the lesion, such as monocytes, macrophages, lymphocytes and others. Nonetheless, at least in the case of validated DM-CGI, the sharp between-group differences in DNA methylation and expression levels suggests that the observed changes involved the majority of cells in AAs, thus including VSMCs and endothelial cells. Another study is currently in progress to identify cell typespecific epigenetic changes in vascular lesions.

\section{Acknowledgements}

This study was funded by DINPO (University of Guanajuato), and the 'Program for the Improvement of the University Professorate' of the Mexican Ministry of Education (PROMEPSEP) (S.Z.). We thank Gertrud Lund for critically reading the manuscript.

\section{References}

1. Gallou-Kabani C, Vigé A, Gross MS and Junien C: Nutriepigenomics: lifelong remodelling of our epigenomes by nutritional and metabolic factors and beyond. Clin Chem Lab Med 45: 321-327, 2007

2. Waterland RA and Jirtle RL: Transposable elements: targets for early nutritional effects on epigenetic gene regulation. Mol Cell Biol 23: 5293-5300, 2003.

3. Lund G, Andersson L, Lauria M, Lindholm M, Fraga MF, Villar-Garea A, Ballestar E, Esteller M and Zaina S: DNA methylation polymorphisms precede any histological sign of atherosclerosis in mice lacking apolipoprotein E. J Biol Chem 279: 29147-29154, 2004.

4. Hiltunen MO, Turunen MP, Hakkinen TP, Rutanen J, Hedman M, Makinen K, Turunen AM, Alto-Setala K and Yla-Herttuala S: DNA hypomethylation and methyltransferase expression in atherosclerotic lesions. Vasc Med 7: 5-11, 2002.

5. Baccarelli A, Tarantini L, Wright RO, Bollati V, Litonjua AA, Zanobetti A, Sparrow D, Vokonas P and Schwartz J: Repetitive element DNA methylation and circulating endothelial and inflammation markers in the VA normative aging study. Epigenetics 5: April 1, 2010 (Epub ahead of print).

6. Baccarelli A, Wright RO, Bollati V, Tarantini L, Litonjua AA, Suh HH, Zanobetti A, Sparrow D, Vokonas PS and Schwartz J: Rapid DNA methylation changes after exposure to traffic particles. Am J Respir Crit Care Med 179: 572-578, 2009.

7. Pogribny IP and Beland FA: DNA hypomethylation in the origin and pathogenesis of human diseases. Cell Mol Life Sci 66: 2249-2261, 2009.

8. Bird A, Taggart M, Frommer M, Miller OJ and Macleod D: A fraction of the mouse genome that is derived from islands of nonmethylated, CpG-rich DNA. Cell 40: 91-99, 1985.

9. Greger V, Passarge E, Höpping W, Messmer E and Horsthemke B: Epigenetic changes may contribute to the formation and spontaneous regression of retinoblastoma. Hum Genet 83: 155-158, 1989.

10. Shann YJ, Cheng C, Chiao CH, Chen DT, Li PH and Hsu MT. Genome-wide mapping and characterization of hypomethylated sites in human tissues and breast cancer cell lines. Genome Res 18: 791-801, 2008.

11. Tusnády GE, Simon I, Váradi A and Arányi T: BiSearch: Primer-design and search tool for PCR on bisulfite treated genomes. Nucleic Acids Res 33: e9, 2005.

12. Bailey TM and Elkan C: Fitting a mixture model by expectation maximization to discover motifs in biopolymers. In: Proceedings of the Second International Conference on Intelligent Systems for Molecular Biology. AAAI Press, Menlo Park, CA, pp28-36, 1994.

13. Previti C, Harari O, Zwir I and del Val C: Profile analysis and prediction of tissue-specific $\mathrm{CpG}$ island methylation classes. BMC Bioinformatics 10: 116, 2009. 
14. Kim J, Kim JY, Song KS, Lee YH, Seo JS, Jelinek J, Goldschmidt-Clermont PJ and Issa JP: Epigenetic changes in estrogen receptor beta gene in atherosclerotic cardiovascular tissues and in-vitro vascular senescence. Biochim Biophys Acta 1772: 72-80, 2007

15. Devlin AM, Singh R, Wade RE, Innis SM, Bottiglieri T and Lentz SR: Hypermethylation of Fads2 and altered hepatic fatty acid and phospholipid metabolism in mice with hyperhomocysteinemia. J Biol Chem 282: 37082-37090, 2007.

16. Zawadzki C, Chatelain N, Delestre M, Susen S, Quesnel B, Juthier F, Jeanpierre E, Azzaoui R, Corseaux D, Breyne J, Torpier G, Staels B, Van Belle E and Jude B: Tissue factor pathway inhibitor-2 gene methylation is associated with low expression in carotid atherosclerotic plaques. Atherosclerosis 204: e4-e14, 2009.

17. Weber M, Hellmann I, Stadler MB, Ramos L, Pääbo S, Rebhan $M$ and Schübeler D: Distribution, silencing and evolutionary impact of promoter DNA methylation in the human genome. Nat Genet 39: 457-466, 2007.

18. Shen L, Kondo Y, Guo Y, Zhang J, Zhang L, Ahmed S, Shu J, Chen X, Waterland RA and Issa J-PJ: Genome-wide profiling of DNA methylation reveals a class of normally methylated CpG island promoters. PLoS Genet 10: 2023-2036, 2007.

19. Ghazi H, Gonzales FA and Jones PA: Methylation of CpGisland-containing genes in human sperm, fetal and adult tissues. Gene 114: 203-210, 1992.

20. Turunen MP, Aavik E and Ylä-Herttuala S: Epigenetics and atherosclerosis. Biochim Biophys Acta 1790: 886-891, 2009.

21. Christensen BC, Houseman EA, Marsit CJ, Zheng S, Wrensch MR, Wiemels JL, Nelson HH, Karagas MR, Padbury JF, Bueno R, Sugarbaker DJ, Yeh RF, Wiencke JK and Kelsey KT: Aging and environmental exposures alter tissue-specific DNA methylation dependent upon $\mathrm{CpG}$ island context. PLoS Genet 5: e1000602, 2009.

22. Gorski DH and Walsh K: Control of vascular cell differentiation by homeobox transcription factors. Trends Cardiovasc Med 6: 213-220, 2003

23. Tommasi S, Karm DL, Wu X, Yen Y and Pfeifer GP: Methylation of homeobox genes is a frequent and early epigenetic event in breast cancer. Breast Cancer Res 11: R14, 2009.

24. Takeshita K, Satoh M, Ii M, Silver M, Limbourg FP, Mukai Y, Rikitake Y, Radtke F, Gridley T, Losordo DW and Liao JK: Critical role of endothelial Notch 1 signaling in postnatal angiogenesis. Circ Res 100: 70-78, 2007.

25. Campagnolo L, Leahy A, Chitnis S, Koschnick S, Fitch MJ, Fallon JT, Loskutoff D, Taubman MB and Stuhlmann H: EGFL7 is a chemoattractant for endothelial cells and is upregulated in angiogenesis and arterial injury. Am J Pathol 167: 275-284, 2005.
26. Shibuya T, Watanabe K, Yamashita H, Shimizu K, Miyashita H, Abe M, Moriya T, Ohta H, Sonoda H, Shimosegawa T, Tabayashi $\mathrm{K}$ and Sato $\mathrm{Y}$ : Isolation and characterization of vasohibin-2 as a homologue of VEGF-inducible endotheliumderived angiogenesis inhibitor vasohibin. Arterioscler Thromb Vasc Biol 26: 1051-1057, 2006.

27. Fish JE, Santoro MM, Morton SU, Yu S, Yeh RF, Wythe JD, Ivey KN, Bruneau BG, Stainier DY and Srivastava D: miR-126 regulates angiogenic signaling and vascular integrity. Dev Cell 15: 272-284, 2008.

28. Thyberg J, Nilsson J, Palmberg L and Sjölund M: Adult human arterial smooth muscle cells in primary culture. Modulation from contractile to synthetic phenotype. Cell Tissue Res 239: 69-74, 1985.

29. Van der Loop FT, Gabbiani G, Kohnen G, Ramaekers FC and van Eys GJ: Differentiation of smooth muscle cells in human blood vessels as defined by smoothelin, a novel marker for the contractile phenotype. Arterioscler Thromb Vasc Biol 17: 665-671, 1997.

30. Shi C, Sakuma M, Mooroka T, Liscoe A, Gao H, Croce KJ, Sharma A, Kaplan D, Greaves DR, Wang Y and Simon DI: Down-regulation of the forkhead transcription factor Foxp1 is required for monocyte differentiation and macrophage function. Blood 112: 4699-4711, 2008.

31. Huttunen HJ, Kuja-Panula J, Sorci G, Agneletti AL, Donato R and Rauvala $\mathrm{H}$ : Coregulation of neurite outgrowth and cell survival by amphoterin and S100 proteins through receptor for advanced glycation end products (RAGE) activation. J Biol Chem 275: 40096-40105, 2000.

32. Yideng J, Jianzhong Z, Ying H, Juan S, Jinge Z, Shenglan W, Xiaoqun $\mathrm{H}$ and Shuren W: Homocysteine-mediated expression of SAHH, DNMTs, MBD2, and DNA hypomethylation potential pathogenic mechanism in VSMCs. DNA Cell Biol 26: 603-611, 2007.

33. Zhang X, Yazaki J, Sundaresan A, Cokus S, Chan SW, Chen H, Henderson IR, Shinn P, Pellegrini M, Jacobsen SE and Ecker JR: Genome-wide high-resolution mapping and functional analysis of DNA methylation in arabidopsis. Cell 126: 1189-1201, 2006.

34. Eckhardt F, Lewin J, Cortese R, Rakyan VK, Attwood J, Burger M, Burton J, Cox TV, Davies R, Down TA, Haefliger C, Horton R, Howe K, Jackson DK, Kunde J, Koenig C, Liddle J, Niblett D, Otto T, Pettett R, Seemann S, Thompson C, West T, Rogers J, Olek A, Berlin K and Beck S: DNA methylation profiling of human chromosomes 6, 20 and 22. Nat Genet 38: 1378-1385, 2006.

35. Khachigian LM: Early growth response-1 in cardiovascular pathobiology. Circ Res 98: 186-191, 2006. 